This item was submitted to Loughborough's Research Repository by the author.

Items in Figshare are protected by copyright, with all rights reserved, unless otherwise indicated.

\title{
Distributed synthetic inertia control in power systems
}

PLEASE CITE THE PUBLISHED VERSION

https://doi.org/10.1109/CIEM.2017.8120874

\section{PUBLISHER}

(C) Institute of Electrical and Electronics Engineers (IEEE)

\section{VERSION}

AM (Accepted Manuscript)

\section{LICENCE}

CC BY-NC-ND 4.0

\section{REPOSITORY RECORD}

Chamorro, Harold R., Andres C. Sanchez, Amund Overjordet, Fernando Jimenez, Francisco M. GonzalezLongatt, and Vijay K. Sood. 2019. "Distributed Synthetic Inertia Control in Power Systems". figshare.

https://hdl.handle.net/2134/32387. 


\title{
Distributed Synthetic Inertia Control in Power Systems
}

\author{
Harold R. Chamorro, Member, IEEE, Andres C. Sanchez, Student Member, IEEE, Amund Øverjordet, Mem- \\ ber, IEEE, Fernando Jimenez, Member, IEEE, Francisco Gonzalez- \\ Longatt, Senior Member, IEEE, and Vijay K. Sood, Senior Member, IEEE
}

\begin{abstract}
Due to the increasing use of renewables into the grid connected through power converters, the rotational inertia in power systems has been reducing. Consequently the frequency response requires the activation of the so-called synthetic inertia control. The synthetic inertia control aims to inject an extra power component when the system experiences a frequency disturbance event. In this paper, it is proposed that a distributed dynamic controllers for sharing the synthetic inertia control actions between the various active power converters in the grid for the improvement of the frequency response. It is assumed that a communication structure between the synthetic inertia controllers and the local power converters is involved in the system. The convergence of the control system is reached through a game population theory and the primary frequency control has been improved. The results are validated based on simulation of a two-area test system.
\end{abstract}

Keywords-Synthetic Inertia Control, Multi-Agents Systems, Population Dynamics, Evolutionary Game Theory, Frequency Response, Non-Synchronous Generation Integration, Renewable Energy Sources, Wind Power.

\section{INTRODUCTION}

$\mathbf{T}$ He current renewable energy targets for 2020 in the European countries have incentivized the various governments to promote the large-scale integration of nonsynchronous generation and deploy several demonstrations of grid enhancement [1]. The integration of non-synchronous generation relies on the use of high power electronics converters for interconnecting and supplying different regions through advanced control capabilities.

One of the challenges of the substantially increased amounts of electricity from non-synchronous generation is the inertia reduction [2], and consequently, the resulting larger excursions experienced in the power system frequency during disturbances. In this fashion, if there is no added a supplementary

Harold R. Chamorro is a PhD student at KTH Royal Institute of Technology, Stockholm, Sweden, e-mail: hr.chamo@ieee.org, hrcv@kth.se.

Andres Sanchez is with University of Los Andes, Bogota, Colombia, email: ac.sanchez11@uniandes.edu.co.

Ammund Øverjordet is with Stannet, Norwegian Transmission System Operator, Oslo, Norway, e-mail: amund.overjordet@statnett.no.

Fernando Jimenez is with University of Los Andes, Bogota, Colombia, email: fjimenez@uniandes.edu.co.

Francisco Gonzalez-Longatt is with Loughborough University, Leicestershire,United Kingdom, e-mail: f.gonzalez-longatt@lboro.ac.uk

Vijay K. Sood is with the Department of Electrical and Computer Engineering of the University of Ontario, Institute of Technology,Ontario, Canada, e-mail: vijay.sood@uoit.ca. control to inject an extra power into the non-synchronous generation control loops [3].

The synthetic inertia control has been formulated and developed by several authors. Authors in [4] explain different activation schemes of synthetic inertia for Full Rated Converters (FRC) in order to improve the frequency response in a test power system.

A comparison of virtual inertia control parameters is presented in [5], and is applied to a multi-area power system where the frequency response is analysed. Additionally, reference [6] presents the effect of wind integration on the system frequency response on the tie-line interchanges in an interconnected system. Simultaneously, the metamorphosis of the power systems into Cyber-Physical Systems (CBS), where remote measurements and control interactions are combined, have brought forth opportunities to propose new control architectures for solving common goals in power systems [7], [8]. Multi-Agent Systems (MAS) based controllers are a mechanism for solving such control problems in a distributed manner.

MAS have been used widely in several power systems applications such as distributed automatic generation control [9], microgrids management [10], distributed Power System Stabilizers (PSS) [11],[12], distributed power injection in HVDC systems [13], distributed dispatch [14], and distributed active power control [15].

One possible MAS method, the population dynamics, which describes how agents can evolve over time [16], and it is inspired by the biological evolution affected by the differential of fitness is applied in this paper. Since generally, the wind power is distributed, and in order to maximize the power injection through the converters action under a disturbance, this paper proposes a distributed synthetic inertia control taking remote information. The strategy uses a set of agents, associated with each Grid Side Converter (GSC) interacting to maximize the total power injected and, improving the primary frequency control in a power system. The system is based on the assumption that the agents are identical and the task is shared between each other.

The paper is organized as follows: In Section II, an introduction to the method used in the paper is presented. Also, a basic introduction to MAS systems and the application of population dynamics is given. Section III covers the power system modeling including a detailed model of a wind farm and its converter current control with synthetic inertia. In Section IV a description of the population dynamics applied to the synthetic inertia control problem is given. The section 
also includes the performance metrics, the cost function and the replicator dynamics equation. Section $\mathrm{V}$ presents the test system used and the results where the improved response of the system frequency through the application of the proposed method is shown. Finally, the conclusions and future work are given.

\section{REPLiCATOR DYNAMiCS PRELIMINARIES}

Fig 1 depicts a MAS, with two different layers. One is the topology of the power systems network, and the second one is the Network Control System (NCS) which applies the distributed control.

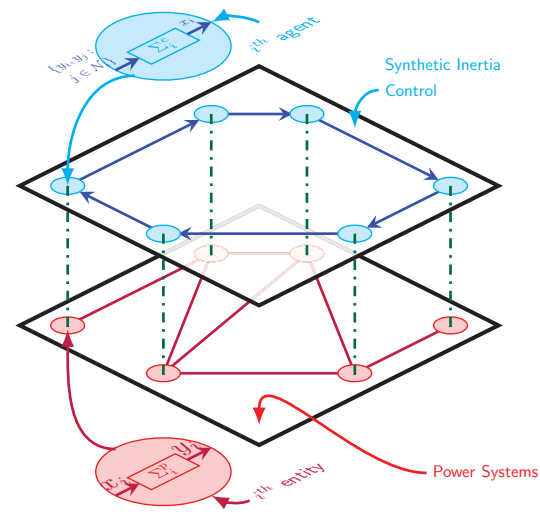

Fig. 1: A large-scale system controlled by a network of agents

The successful strategy $i^{t h}$ is generally adopted by most agents. These agents are a population $x_{i}$ that will grow according to fitness function $f_{i}$. This growth is related to the parameter $\beta>0$.

$$
\dot{x}_{i}=\beta x_{i}\left(f_{i}-\bar{f}\right)
$$

The total population $P$ and the average fitness $\bar{f}$ are given by:

$$
P=\sum_{i=1}^{N} x_{i} \quad \bar{f}=\frac{1}{P} \sum_{i=1}^{N} x_{i} \cdot f_{i}
$$

\section{POWER System Modelling}

\section{A. Swing Equation}

A power system can be conveniently described by a diagram consisting of a set of edges and vertices. An undirected graph $\mathcal{G}=(\mathcal{V}, \mathcal{E})$ is used to represent an electrical network modeled by $n_{b}$ buses, denoted by the set of network nodes by $\mathcal{V}=\left\{1, \cdots, n_{b}\right\}$, with a subset of $g$ machines, $\mathcal{E}$ is the set of power transmission lines, and the voltages at these buses $\bar{U}_{g}$. We consider a heterogeneous network with three distinct sets of nodes $\mathcal{V}=\mathcal{G} \cup \mathcal{F} \cup \mathcal{P}$, corresponding to buses connecting synchronous generators, non-synchronous generators and loads. Synchronous generators are connected at $n_{b}>g \geq 1$ nodes $\mathcal{G}=\{1, \ldots, g\}$, the set of non-synchronous generators network nodes is given by $\mathcal{F}=\{g+1, \ldots, g+f\}$, where $n_{b}>f \geq 0$, and the set of loads are connected at $\mathcal{P}=$ $\{g+f+1, \ldots, n\}$, where $n_{b}>p \geq 0$.

The dynamic of the $i^{t h}$ synchronous generator is given by [17]:

$$
\dot{\omega}_{i}=\frac{1}{2 H_{i}}\left(P_{i}^{m}-P_{i}^{e}-D_{i} \omega_{i}\right), i \in \mathcal{G}
$$

where $\omega_{i}($ in $\mathrm{rad} / \mathrm{s})$ is the rotor speed of generator $i, P_{i}^{m}$ is the mechanical power (p.u.), $P_{i}^{e}$ is the electric power (p.u.), $H_{i} \in \mathbb{R}_{>0}$ is the inertia constant and $D_{i} \in \mathbb{R}_{>0}$ is the droop damping.

The Frequency Containment Response (FCR) is automatically enforced by speed governors, implemented at conventional generator units. This governor relates the generator output power to a reference power and the frequency deviation present in the system. This change in output power follows the governors droop.

\section{B. Non-synchronous Generation and Synthetic Inertia}

Following (3), the dynamics of the system frequency are written as:

$$
2 H_{i} \dot{\omega}_{i}=P_{i}^{m}-P_{i}^{e}+P_{i}^{n s}-D_{i} \omega_{i}
$$

A higher aggregate inertia helps the system to remain stable and reduces the rate of change in angular speed. The inertial enhancement control from the non-synchronous generation $P_{i}^{n s}$ is:

$$
P_{i}^{n s}=P_{i}^{\text {ord }}-x_{i} \dot{\omega}_{i}, i \in \mathcal{F}
$$

where, $x_{i}$ is the synthetic inertia regulator and $P_{i}^{\text {ord }}$ is the ordinary power supplied by the non-synchronous generation [18]. Hence, resulting in the following dynamics of the system frequency:

$$
\left(2 H_{i}+x_{i}\right) \dot{\omega}_{i}=P_{i}^{m}-P_{i}^{e}+P_{i}^{o r d}-D_{i} \omega_{i}
$$

\section{Wind Farm Aggregated Model}

Fig. 2 shows a simplified block diagram of the active power control of a WTG model [22].

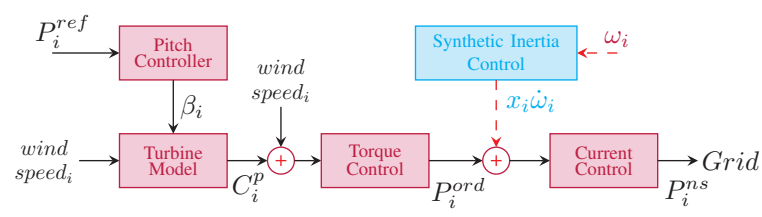

Fig. 2: Wind Farm Structure

The mechanical power that can be extracted from the generator shaft of a wind turbine [22]:

$$
P_{i}^{t}=\frac{1}{2} \rho_{i} A_{i} v_{i}^{3} C_{i}^{p}\left(\lambda_{i}, \mu_{i}\right), i \in \mathcal{F}
$$

where, $\rho_{i}$ is the air density, $A_{i}$ is the area swept by the rotor blades, $v_{i}$ is the wind speed, $\mu_{i}$ denotes pitch angle, and $C_{i}^{p}$ is a function of the pitch angle of the turbine blade. The tip speed ratio $\lambda_{i}$ is defined as: 


$$
\lambda_{i}=\frac{\omega_{i}^{t} R_{i}}{v_{i}}, i \in \mathcal{F}
$$

with $\omega_{i}^{t}$ and $R_{i}$ as the shaft speed and rotor radium respectively. The inputs are the incoming wind, the electrical power injected by the WTG $P_{g}$, and the power set point $P_{\text {ord }}$ which is dominated by the current control injection.

A speed controller regulates the torque. General approaches use the rotational speed of the turbine as an input signal. Thereby, the reference value is set for the torque. In case the electric torque is larger than the mechanical torque, the electric set point will decrease and the turbine will decelerate.

The mechanical speed regulation in the power limiting region is implemented by controlling the blade pitch angle $\mu_{i}$. The control signal $u_{\mu_{i}}$ is given by the following PID control scheme [23]:

$$
\left.\begin{array}{rl}
u_{\mu_{i}} & =-C_{i}^{p t}(s)\left(\omega_{i}^{r e f}-\omega_{i}^{m}\right) \\
C_{i}^{p t}(s) & =k_{i}^{p p}\left(1+\frac{1}{T_{i}^{i} s}+\frac{T_{i}^{d} s}{1+\gamma_{i} T_{i}^{d} s}\right)
\end{array}\right\}: i \in \mathcal{F}
$$

where, $\omega_{i}^{r e f}$ is the reference for the regulation, and $k_{i}^{p p}, T_{i}^{i}$, $T_{i}^{d}$ and $\gamma_{i}$ denote control parameters.

The main purpose of the synthetic inertia controller $x_{i}$ is to improve the instantaneous frequency deviation. The active damping approach is applied to the current control to improve the power injection response.

Fig. 3 shown the current control. The conventional $d q$ current control has been widely studied in the literature.

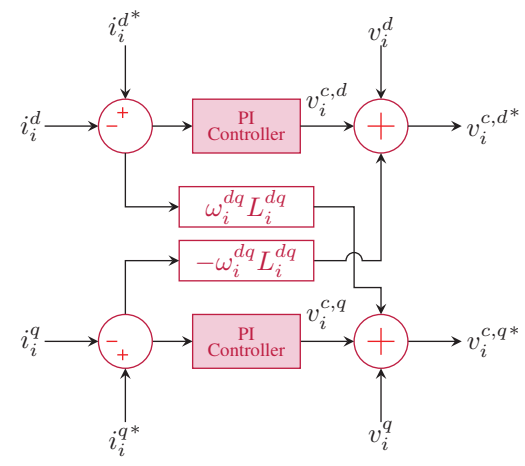

Fig. 3: Current Control blocks

The dynamics of the $d-q$ axes are:

$$
i \in \mathcal{F}:\left\{\begin{array}{l}
R_{i}^{d q} i_{i}^{d}+L_{i}^{d q} \frac{d i_{i}^{d}}{d t}=v_{i}^{c, d}+\omega_{i}^{d q} L_{i}^{d q} i_{i}^{q}-v_{i}^{d} \\
R_{i}^{d q} i_{i}^{q}+L_{i}^{d q} \frac{d i_{i}^{q}}{d t}=v_{i}^{c, q}-\omega_{i}^{d q} L_{i}^{d q} i_{i}^{d}-v_{i}^{q}
\end{array}\right.
$$

The inverter decoupled voltage control of $i_{i}^{d}$ and $i_{i}^{q}$ is noted as follows:

$$
i \in \mathcal{F}:\left\{\begin{array}{l}
v_{i}^{c, d}=u_{i}^{c, d}-L_{i}^{d q} \omega_{i}^{d q} i_{i}^{q}+v_{i}^{d} \\
v_{i}^{c, q}=u_{i}^{c, q}+L_{i}^{d q} \omega_{i}^{d q} i_{i}^{d}+v_{i}^{q}
\end{array}\right.
$$

where, $u_{i}^{c, d}$ and $u_{i}^{c, q}$ are control signals of the $d$ and $q$ axes respectively.

$d$-axis and $q$-axis references are the inner controllers references for the active and the reactive power respectively. The direct and quadrature axes are coupled via coupling terms $\omega_{i}^{d q} L_{i}^{d q} i_{i}^{d}$ and $\omega_{i}^{d q} L_{i}^{d q} i_{i}^{q} . R_{i}^{d q}$ and $L_{i}^{d q}$ stands for the equivalent combined resistance and inductance of the phase reactor and transformer.

Active and reactive power converter currents are controlled in the synchronous reference frame with standards PI controllers. From (10), the plant for the current control is given by:

$$
G_{i}(s)=\frac{I_{i}^{d}(s)}{V_{i}^{d^{\prime}}(s)}=\frac{I_{i}^{q}(s)}{V_{i}^{q^{\prime}}(s)}=\frac{1}{L_{i}^{d q} s+R_{i}^{d q}}, i \in \mathcal{F}
$$

Then,

$$
i \in \mathcal{F}:\left\{\begin{array}{l}
P_{i}^{n s}=v_{i}^{d} i_{i}^{d} \\
Q_{i}^{n s}=-v_{i}^{d} i_{i}^{d}
\end{array}\right.
$$

\section{Synthetic Inertia Management}

Here, it is presented a synthetic inertia control protocol for power systems. Figure 4 shown the two area test system network including two wind farms and the NCS.

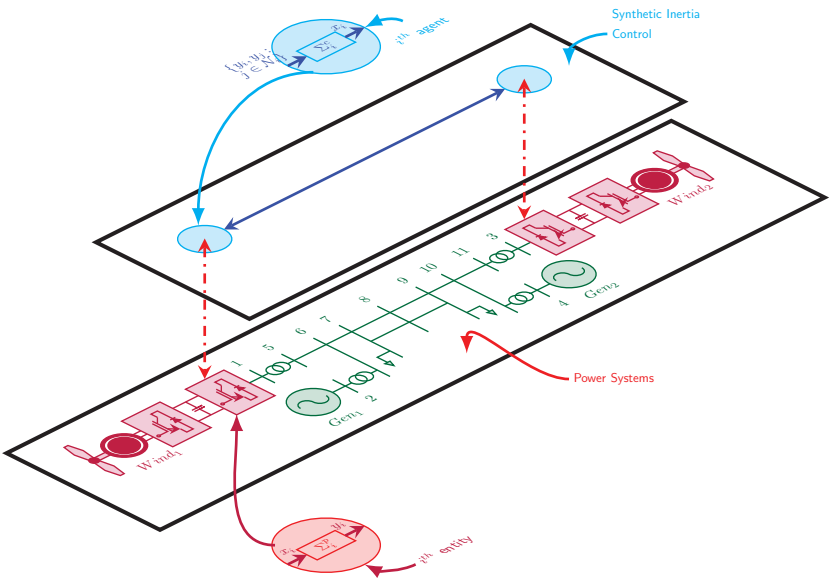

Fig. 4: Network Control System with a Power System

This approach considers a control network system which provides the distributed control signals $x_{i}$ in each GSC. Moreover, there is a connecting graph of the control network. It is supposed full remote measurements and local information.

\section{A. Measurement Metrics}

In order to have an estimated measurement of the frequency of an interconnected system is the CoI (Center of Inertia), which is computed based on the individual speeds $\omega_{i}$ and the inertia constants of the synchronous generators $H_{i}$.

Assuming the set $\mathcal{G}$ of synchronous generators, (14) compute the CoI, and (15) the Rate of Change of Frequency (RoCoF) measurement in the CoI reference is defined. 


$$
\omega_{C o I}=\frac{\Sigma_{i \in \mathcal{G}} H_{i} \omega_{i}}{\Sigma_{i \in \mathcal{G}} H_{i}}
$$

$$
\dot{\omega}_{C o I}=\frac{\Sigma_{i \in \mathcal{G}} H_{i} \frac{d \omega_{i}}{d t}}{\Sigma_{i \in \mathcal{G}} H_{i}}
$$

\section{B. Cost Function}

The following function is proposed:

$\max u(x): \Sigma_{i \in \mathcal{G}}\left(a_{i} \dot{\omega}_{i}+b\right) x_{i}, a_{i} \in \mathbb{R}_{<0}$

subject to $g_{1}(x): \Sigma_{i \in \mathcal{G}} x_{i}=1$

$$
g_{2}(x): x_{i} \geq 0
$$

where $a_{i}$ is the $i^{t h}$ parameter to be tuned in $i^{\text {th }}$ power system. $\dot{\omega}_{i}$ owned information about RoCoF, and $b>0 . u(\cdot)$ is cost function and stricty concave, and $g_{1}(\cdot)$ is the constraint related with proportional action in each power system.

\section{Replicator Dynamics}

The fitness function $f_{i}(\cdot)$ is always positive because $b>0$ and $u(\cdot)$ is strictly concave. Remember, $a_{i} \in \mathbb{R}_{<0}$.

$$
f_{i}\left(\omega_{i}\right)=\frac{\partial u(x)}{\partial x_{i}}=a_{i} \dot{\omega}_{i}+b, \text { for } i=1, \ldots, N
$$

The derivative term in the fitness function provides information about RoCoF. Therefore, in case of a strong change in $\omega_{i}$, the controller will apply a signal $x_{i}$ to avoid undershoots in accordance with the derivative action.

Fig. 5 shows feedback interconnected between Power System $\Sigma^{p}$ and Synthetic Inertia Control via Replicator Dynamics $\Sigma^{c}$.

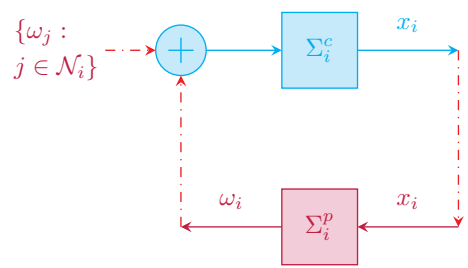

Fig. 5: Interconnected Systems $\Sigma_{i}^{c}$ and $\Sigma_{i}^{p}$

This interconnection is modeled by (18)

$$
\begin{aligned}
& \Sigma^{p}:\left\{\begin{array}{l}
\dot{\omega}_{i}=\frac{1}{2 H_{i}+x_{i}}\left(P_{i}^{m}-P_{i}^{e}+P_{i}^{\text {ord }}-D_{i} \omega_{i}\right) \\
y_{1 i}=\omega_{i}
\end{array}\right. \\
& \Sigma^{c}:\left\{\begin{array}{l}
\dot{x}_{i}=\beta\left(x_{i}+\omega_{C o I}\right)\left(f_{i}-\sum_{j=1}^{N} f_{j}\left(x_{j}+\omega_{C o I}\right)\right) \\
y_{2 i}=-x_{i}
\end{array}\right. \\
& \text { for } i=1, \ldots, N
\end{aligned}
$$

where $x_{i}=\omega_{i}-\omega_{C o I} . x_{i}$ is defined based on Fig. 5 as an output of $\Sigma^{c}$ by adjusting (14) in (1). Note that due to $g_{1}(\cdot)$ and $g_{2}(\cdot)$ (simplex), the derivative action of RD-based method is kept within a safety margin. Then, derivative term is used to damp the undershoot while control effort is constrained by the simplex.

\section{System Description And Results}

\section{A. Test System}

The test system (Fig.6) is composed symmetricallly by two areas connected to each other via two $230 \mathrm{kV}$ lines of $220 \mathrm{~km}$ length [24]. Round rotor generators are rated at $20 \mathrm{kV} / 900$ MVA. $50 \mathrm{~Hz}$ is taken as nominal power system frequency. In order to test the method proposed, two of the generators have been replaced by non-synchronous generation of an aggregated wind farm model.

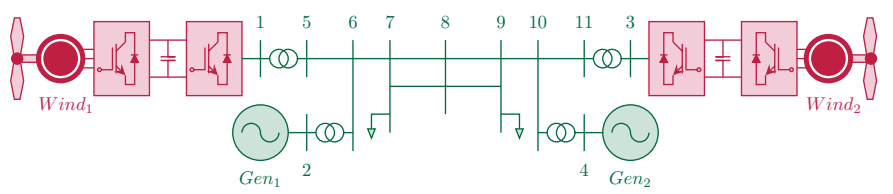

Fig. 6: Two-area Test System

\section{B. Simulation Results}

The impact of the proposed distributed controller is analysed through time-domain simulations carried out in Matlab/Simulink. The frequency response is evaluated during an increase in the load of $600 \mathrm{MW}$ at bus 9 at $t=100 \mathrm{~s}$. Note, it is assumed that a direct communication link between the units.

Since the non-synchronous generation units are identical, an slight parameters modification has been done in one them in order to observe the convergence and the difference in the distributed control actions by each one. Otherwise, an identical response will be given. Fig. 7 shows the frequency response in the CoI reference frame with and without the action of the distributed synthetic inertia control by both of the nonsynchronous generation units. A clear improvement in the frequency response has been reached. The frequency nadir without the synthetic inertia control loop reaches $f_{\min }=48.51$ $\mathrm{Hz}$, whereas with the proposed method reaches $f_{\min }=48.98$ $\mathrm{Hz}$.

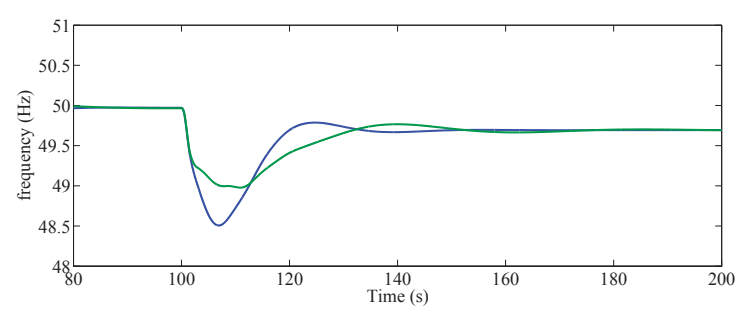

Fig. 7: Frequency Response of the Test System

Frequency response and power injection of each nonsynchronous generation unit is shown in Figures $8 \mathrm{a}$ and $8 \mathrm{~b}$ respectively. Both figures show the similarity in the control action and the convergence by the proposed method.

\section{CONCLUSIONS}

The increasing use of converter-fed renewable sources into the power system has reduced the rotating inertia available 


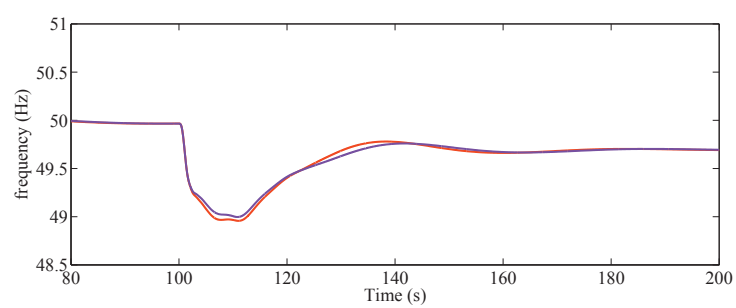

(a) Frequency Response of the Individual agents

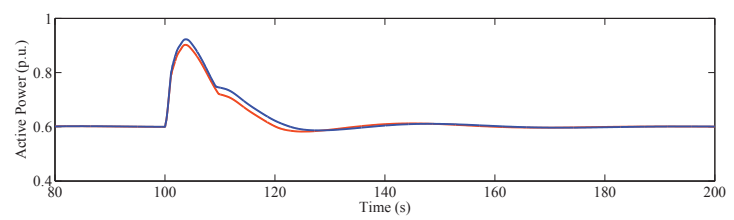

(b) Power Delivered by Each Converter

Fig. 8: Individual Agents Response

with the consequence that dynamic frequency control is being affected. The introduction of a synthetic inertia control input within the converter controllers offers a counter measure to correct the actual lack of rotational inertia. Since the grid side converters are widely distributed within the power system, in this paper, a distributed control system for the management of the synthetic inertia control has been proposed. A game theory strategy has been used to distribute dynamically the control actions to the grid converters. A communication system is assumed to coordinate the actions of the various controllers. In the two-area test system used, the frequency response has been improved significantly compared to the case without the distributed synthetic control. As a future work, it is intended to apply the same method in a larger power system in order to verify the proposed distributed strategy. The impact of any telecommunication delays will also be studied.

\section{REFERENCES}

[1] M. Lorenzo, U. Burdalo, R. Veguillas, A. Birke, O. Despouys, I. P. Byriel, C. Druet, A. Abart, G. Bissel, and M. Sebastian-Viana, "The Proof Is in the Putting: Large-Scale Demonstrations of Renewables Integration Showcase Real-World Solutions," IEEE Power and Energy Magazine, vol. 13, pp. 75-83, Jan. 2015.

[2] W. Winter, K. Elkington, G. Bareux, and J. Kostevc, "Pushing the Limits: Europe's New Grid: Innovative Tools to Combat Transmission Bottlenecks and Reduced Inertia," IEEE Power and Energy Magazine, vol. 13, pp. 60-74, Jan. 2015.

[3] E. Rakhshani, D. Remon, A. M. Cantarellas, and P. Rodriguez, "Analysis of derivative control based virtual inertia in multi-area high-voltage direct current interconnected power systems," Transmission Distribution IET Generation, vol. 10, no. 6, pp. 1458-1469, 2016.

[4] F. Gonzalez-Longatt, "Activation schemes of synthetic inertia controller on full converter wind turbine (type 4)," in 2015 IEEE Power Energy Society General Meeting, pp. 1-5, July 2015.

[5] Z. Zhang, Y. Wang, H. Li, and X. Su, "Comparison of inertia control methods for DFIG-based wind turbines," in 2013 IEEE ECCE Asia Downunder, pp. 960-964, June 2013.
[6] N. Nguyen and J. Mitra, "An Analysis of the Effects and Dependency of Wind Power Penetration on System Frequency Regulation," IEEE Transactions on Sustainable Energy, vol. 7, pp. 354-363, Jan. 2016.

[7] F. L. Lewis, Cooperative control of multi-agent systems: optimal and adaptive design approaches. Amsterdam: Springer, 2014. OCLC: 891508597.

[8] A. W. Colombo, S. Karnouskos, O. Kaynak, Y. Shi, and S. Yin, "Industrial Cyberphysical Systems: A Backbone of the Fourth Industrial Revolution,' IEEE Industrial Electronics Magazine, vol. 11, pp. 6-16, Mar. 2017.

[9] Z. Li, C. Zang, P. Zeng, H. Yu, and H. Li, "MAS based distributed automatic generation control for cyber-physical microgrid system," IEEE/CAA Journal of Automatica Sinica, vol. 3, pp. 78-89, Jan. 2016.

[10] H. R. Chamorro and N. L. Díaz, "Hierarchical power flow control in low voltage microgrids," in 2013 North American Power Symposium (NAPS), pp. 1-5, Sept. 2013.

[11] F. Dörfler, M. R. Jovanović, M. Chertkov, and F. Bullo, "SparsityPromoting Optimal Wide-Area Control of Power Networks," IEEE Transactions on Power Systems, vol. 29, pp. 2281-2291, Sept. 2014.

[12] H. Chamorro and M. Rios, "Hierarchical PSS control for smart transmission grids - A multiagents system approach," in PowerTech (POWERTECH), 2013 IEEE Grenoble, pp. 1-5, June 2013.

[13] D. Babazadeh, D. Van Hertem, and L. Nordström, "Study of centralized and distributed coordination of power injection in multi-TSO HVDC grid with large off-shore wind integration," Electric Power Systems Research, vol. 136, pp. 281-288, July 2016.

[14] A. Pantoja and N. Quijano, "A Population Dynamics Approach for the Dispatch of Distributed Generators," IEEE Transactions on Industrial Electronics, vol. 58, pp. 4559-4567, Oct. 2011.

[15] G. Chen, F. L. Lewis, E. N. Feng, and Y. Song, "Distributed Optimal Active Power Control of Multiple Generation Systems," IEEE Transactions on Industrial Electronics, vol. 62, pp. 7079-7090, Nov. 2015.

[16] J. W. Weibull, Evolutionary game theory. Cambridge, Mass. [u.a.: MIT Press, 2004. OCLC: 255505178 .

[17] P. Kundur, N. J. Balu, and M. G. Lauby, Power system stability and control. New York: McGraw-Hill, 1994. OCLC: 28929603.

[18] H. R. Chamorro, C. A. Ordonez, J. C. Peng, and M. Ghandhari, "Non-synchronous generation impact on power systems coherency," Transmission Distribution IET Generation, vol. 10, no. 10, pp. 2443 2453, 2016.

[19] G. Lalor, A. Mullane, and M. O'Malley, "Frequency control and wind turbine technologies," IEEE Transactions on Power Systems, vol. 20, pp. $1905-1913$, Nov. 2005.

[20] Y. Wang, G. Delille, H. Bayem, X. Guillaud, and B. Francois, "High wind power penetration in isolated power systems - assessment of wind inertial and primary frequency responses," IEEE Transactions on Power Systems, vol. 28, no. 3, pp. 2412-2420, 2013.

[21] D. Banham-Hall, C. Smith, G. Taylor, and M. Irving, "Investigating the limits to inertial emulation with large-scale wind turbines with direct-drive permanent magnet generators," in UKACC International Conference on Control 2010, pp. 1-6, Sept. 2010.

[22] H. Dharmawardena, K. Uhlen, and S. S. Gjerde, "Modelling wind farm with synthetic inertia for power system dynamic studies," in 2016 IEEE International Energy Conference (ENERGYCON), pp. 1-6, Apr. 2016.

[23] N. Horiuchi and T. Kawahito, "Torque and power limitations of variable speed wind turbines using pitch control and generator power control," in Power Engineering Society Summer Meeting, 2001, vol. 1, pp. 638-643 vol.1, July 2001.

[24] M. Klein, G. Rogers, and P. Kundur, "A fundamental study of inter-area oscillations in power systems," IEEE Transactions on Power Systems, vol. 6, pp. 914-921, Aug. 1991. 